\title{
Synaptonemal complexes in female and male meiotic prophase of Ephestia kuehniella (Lepidoptera)
}

\author{
FRANTIŠEK MAREC* \& WALTHER TRAUT† \\ *Institute of Entomology, Czech Academy of Sciences, Branišovská 31, CZ-37005 Ceské Budějovice, Czech Republic; \\ $\dagger$ †nstitut für Biologie der Medizinischen Universität zu Lübeck, Ratzeburger Allee 160, D-2400 Lübeck 1, Germany
}

\begin{abstract}
Synaptonemal complex (SC) complements of oocyte and spermatocyte pachytene nuclei in the Mediterranean flour moth, Ephestia kuehniella, were investigated using a microspreading technique and electron microscopy. In both sexes, three autosomal SCs could be identified by their morphological landmarks; one SC has a prominent knob and two SCs have nucleolus organizer regions. In a later stage of SC development in oocytes, the two nucleolar SCs displayed electron dense terminal modifications of the lateral elements. In some oocytes, the sex chromosome bivalent was inconspicuous but in the majority it was recognized by heterochromatin tangles associated with the $\mathrm{W}$ chromosome, a shorter $\mathrm{W}$ chromosome axis and/or incomplete pairing. Male synaptonemal complexes in contrast to female ones contained recombination nodules (RNs). Almost every bivalent was associated with one RN, rarely none or two RNs. Along the SCs, RNs were preferentially located in distal positions. The presence of RNs in male SCs and their absence in female SCs corresponds with the presence of crossing over and chiasmata in male meiosis and their absence in female meiosis. The mean total length of SC complements was greater in males than in females, but in both sexes, variation was considerable. We assume that male SCs become longer with progression of the pachytene stage while in female SCs a period of elongation is followed by a period of shortening.
\end{abstract}

Keywords: Mediterranean flour moth, meiosis, microspreading, recombination nodules, synaptonemal complexes.

\section{Introduction}

Sex-specific differences in the course of meiotic prophase in various organisms can frequently be derived from the sex chromosome determining mechanism and from the occurrence of crossing over. In this respect, most species of the genus Drosophila represent a typical example. Drosophila females, which are the homogametic sex, exhibit the normal sequence of meiotic events. The heterogametic Drosophila males lack crossing over. Correspondingly, their meiosis is achiasmatic and synaptonemal complexes (SCs) which are thought to be a prerequisite for recombination are not formed at all (see John, 1990).

An essentially similar but reverse meiotic situation is known from insects of the order Lepidoptera. Butter-

Correspondence: František Marec, Institute of Entomology, Czech Academy of Sciences, Branišovská 31, CZ-370 05 České Budějovice, Czech Republic. flies and moths, with few exceptions, possess a WZ/ZZ chromosome mechanism of sex determination (Suomalainen, 1969a; Robinson, 1971). Females are the heterogametic sex displaying achiasmatic meiosis (Suomalainen, 1969b; Traut, 1977). During oogenesis, eight sister germ cells are connected in a cluster and develop in parallel up to the pachytene stage. In contrast to Drosophila, all cells of the cluster form SCs (Telfer, 1975). After pachytene, seven cells are transformed into nurse cells and only one oocyte in a cluster completes meiosis. The SCs of the oocyte do not disintegrate but are transformed into modified SCs by shortening of the bivalents and retained to metaphase I (Rasmussen, 1976; Traut, 1977; Rasmussen \& Holm, 1982). According to Rasmussen (1977), these modified SCs serve as chiasma substitutes. Male meiosis on the other hand is chiasmatic with a normal sequence of meiotic events including the process of recombination (Traut, 1977; Holm \& Rasmussen, 1980). 
At the electron microscopy (EM) level, meiotic prophase has mainly been studied in two lepidopteran species, the silkworm (Bombyx mori) and the Mediterranean flour moth (Ephestia kuehniella). In Bombyx, extensive data have been obtained on the formation of SCs and ultrastructure of meiotic prophase events in both sexes by means of three dimensional reconstruction of serially sectioned nuclei (reviewed by Rasmussen \& Holm, 1982) and also by surface spreading (Rasmussen, 1986). Rattner et al. (1980, 1981) described changes of the chromatin organization in male meiotic nuclei of Bombyx prepared by the Miller spreading procedure. A similar microspreading technique was used by Weith \& Traut $(1980,1986)$ to investigate the SC complement in Ephestia. Their studies were focused on the fine structure of the W chromosome heterochromatin and the pairing behaviour of sex chromosomes in the wild-type and $\mathrm{W}$ chromosome mutant females.

In this paper, we use the microspreading technique for a comparative study of male and female SCs in $E$. kuehniella and their relationship with chiasmatic male and achiasmatic female meiosis.

\section{Materials and methods}

\section{Insects}

A wild-type strain ' $\mathrm{C}$ ' (kept in České Budějovice, for its origin see Marec, 1990) and 12 sex-chromosome mutant lines isolated by Marec \& Mirchi (1990) of $E$. $k u e h n i e l l a$ were used in the present study. The animals were reared on oat flakes at $20-21^{\circ} \mathrm{C}$. For EM preparations, ovaries were dissected from both the feeding and wandering stages of fifth instar larvae and from prepupae. Testes were investigated from third and fourth instar larvae.

\section{Microspreading of pachytene nuclei}

Gonads were dissected out in a saline solution (Glaser, 1917). The unfixed gonads were transferred to a hypotonic solution (83 $\mathrm{mm} \mathrm{KCl}$ and $17 \mathrm{~mm} \mathrm{NaCl}$ ) and disrupted manually using fine tungsten needles. After 10-20 min the swollen material was transferred to a spreading solution with the aid of a micropipette. The spreading solution consisted of 0.02 per cent Joy detergent (Procter and Gamble, U.S.A.) in bidistilled water, adjusted to $p \mathrm{H}$ 8.6-8.9 with $0.01 \mathrm{~m}$ borate buffer solution $(p \mathrm{H}$ 9.22, Merck). Nuclei were allowed to disperse for 5-10 min. Subsequently the material was centrifuged (2200 r.p.m., $\left.4^{\circ} \mathrm{C}, 10 \mathrm{~min}\right)$ in a Teflon microcentrifugation chamber onto hydrophilized EM grids through a $0.1 \mathrm{~m}$ sucrose cushion containing 1 per cent formaldehyde $(p \mathrm{H} \mathrm{8.2-8.5).} \mathrm{Then} \mathrm{the} \mathrm{specimens}$ were fixed for 2 min with fresh formaldehyde-sucrose solution, rinsed for $30 \mathrm{~s}$ in 0.4 per cent detergent (Kodak Foto Flo, $p \mathrm{H} \mathrm{8.0-8.2)} \mathrm{and} \mathrm{air} \mathrm{dried.} \mathrm{Staining}$ was performed in 1 per cent ethanolic phosphotungstic acid for $30 \mathrm{~s}$. Micrographs were taken in a Philips EM 400 transmission electron microscope operated at 80 $\mathrm{kV}$.

\section{Length measurements of SCS}

We selected complete sets of SCs with all 30 bivalents clearly visible for length measurements. Exact magnification of the EM was determined using a calibration grating. EM micrographs were enlarged to a final magnification of $\times 6300$. Lateral elements (LEs) were measured and their lengths calculated using a digitizer tablet and two computer programs, MESSCHRO and EVALChro (written by W. Traut). Pachytene complement lengths are usually expressed as SC lengths. In this study, complement length is the total length of all 60 LEs to accommodate the asymmetrical WZ bivalents in oocyte nuclei.

\section{Results}

\section{Female meiosis}

Separation of nuclei from sibling clusters represented the main technical problem in the preparation of microspreads from oocytes. Despite the mechanical manipulation, detergent treatment and centrifugation, sister nuclei tended to stick together in clusters thus making the evaluation of individual chromosome complements difficult. A cluster of eight sister nuclei is shown in Fig. 1. All nuclei appear in the same pachytene substage, as judged from the appearance of SCs. The synchronized development of oocytes within a cluster until the end of pachytene appears to be a typical feature of Lepidoptera oogenesis (Telfer, 1975; King \& Büning, 1985). In the middle of the cluster, a branched structure decorated with seven electron dense rings is visible. This structure was proved in another study (Marec et al., 1993) to be the polyfusome which is known in higher insects as a continuous structure joining sister gametocytes through a system of intercellular bridges (King et al., 1982). The seven rings regularly arranged in the polyfusome are remnants of the contractile canal rims which stabilize the bridges (Marec et al., 1993).

Although several tens of sibling clusters were screened in the EM and a few of them were examined in detailed micrographs, morphological differences among pachytene complements within a cluster were 


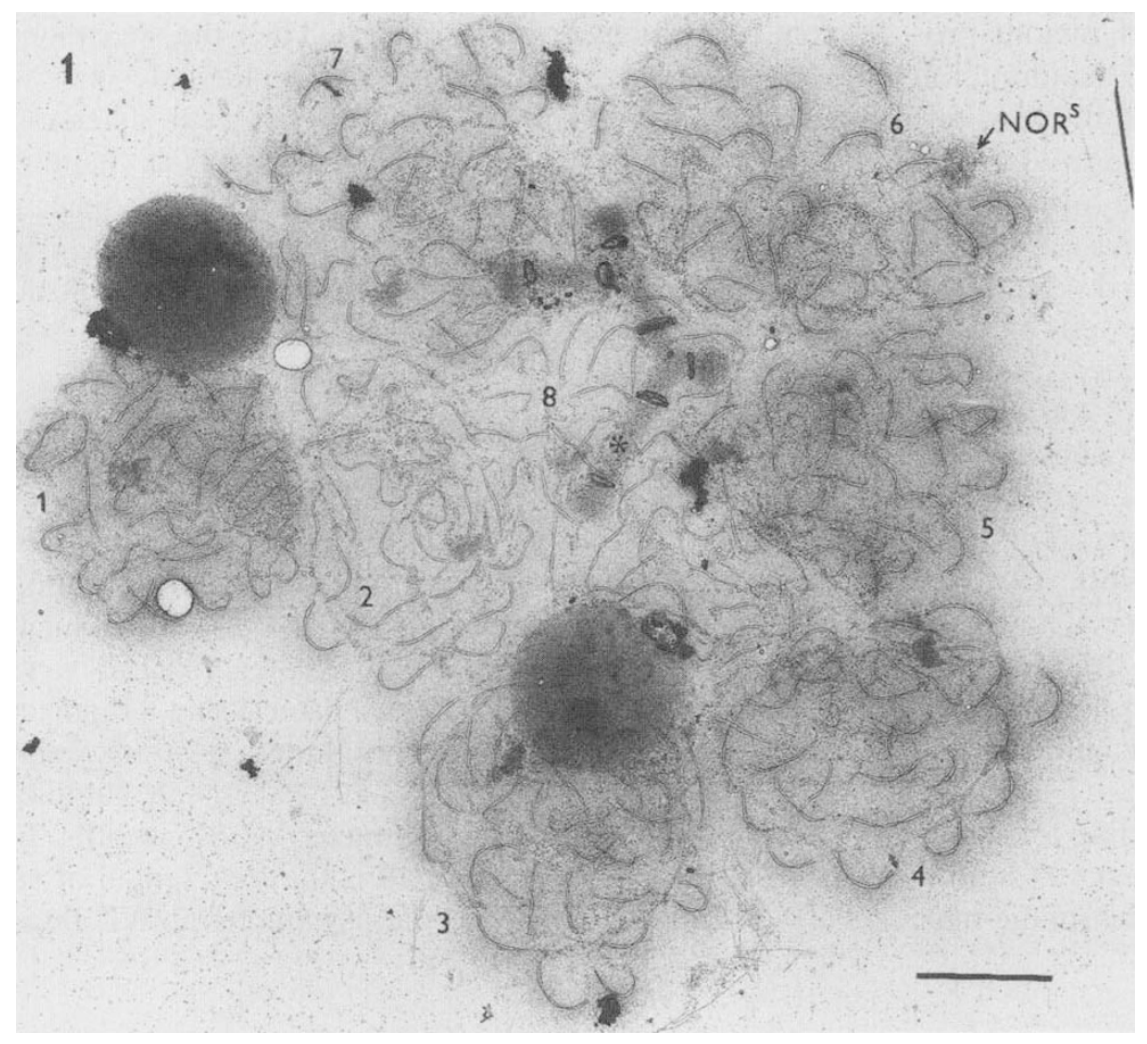

Fig. 1 A cluster of eight sister pachytene oocyte nuclei [ASF-4 line]. The nuclei, which are numbered from 1 to 8 , consist of well spread SCs. However, individual SC complements are not sufficiently separated from each other. In each nucleus, a short bivalent showing the nucleolus organizer region $\left(\mathrm{NOR}^{S}\right)$ can be recognized although it is only marked in complement no. 6 . The branched structure with seven electron dense rings is a polyfusome (asterisk). Two big densely staining spheres represent interphase nuclei which were randomly deposited on the cluster by centrifugation. $\mathrm{Bar}=10 \mu \mathrm{m}$. not found. There is no indication from the SCs that the oocyte nucleus is differentiated from the nuclei of nurse cells in the pachytene stage. This is in accordance with results from EM serial section analyses of oocytes of three lepidopteran species, E. kuehniella (Guelin, 1975), Hyalophora cecropia (Mandelbaum, 1980), and B. mori (Rasmussen \& Holm, 1982).

In well spread pachytene nuclei of wild-type females, the complete chromosome set of 30 bivalents, 29 formed by autosomal homologues and one by the sex chromosomes W and Z, was observed (Fig. 2). Most pachytene nuclei have fully paired autosomal bivalents with typical SCs consisting of two parallel lateral elements (LEs) of equal lengths surrounded by a predominantly uniform halo of chromatin loops. In some preparations, a central element $(\mathrm{CE})$ was visible in the free space between the two LEs (Fig. 6). As Ephestia chromosomes have no localized centromere (Traut, 1986) we did not expect and did not find centromere regions in the SCs.

In some oocytes, the WZ bivalent was inconspicuous but in the majority it was recognized according to the criteria described by Weith \& Traut $(1980,1986)$. In optimally spread preparations, the axis of the W chromosome was decorated with remnants of heterochromatin tangles, discernible by their higher electron density compared with the $\mathrm{Z}$ chromosomal or auto- somal chromatin loops (Figs 2 and 3). The W chromosome LE was mostly perceptibly shorter (on average roughly 20 per cent) than that of the $\mathrm{Z}$ chromosome. However, some fully synapsed sex chromosome SCs displayed only minor differences in the length of their $\mathrm{Z}$ and $\mathrm{W}$ LEs. Taken together with cases in which we failed to identify the WZ bivalent, there is indication that the length difference between LEs of the structurally heterozygous bivalent can be reduced by synaptic adjustment (see Weith \& Traut, 1986). Of the 49 wild-type oocyte complements investigated, 30 (61 per cent) showed complete pairing of the WZ bivalent. Due to their unequal LE lengths, these WZ SCs displayed a characteristic twisting of the longer $\mathrm{Z}$ axis along the shorter $\mathrm{W}$ axis (Fig. 3). In the remaining complements, the WZ bivalent was only partially paired or even not paired at all ( $c f$. Traut et al., 1986). The characteristic features of the WZ bivalents were also observed in females of 12 sex-chromosome mutant lines and used to define structural rearrangements of the sex chromosomes. The specific results on these lines are described elsewhere (Marec \& Traut, 1993). The complete pachytene set was measured in 32 nuclei of wild-type females, the WZ bivalent was recognized in 30 of them. The WZ bivalent was the 1st and 12th longest bivalent. On average the $\mathrm{WZ}$ bivalents represented $3.8 \pm 0.3$ per cent of the total diploid comple- 
Figs 2-5: 2 Female synaptonemal complex complement [wild-type strain]. A total of 30 bivalents is visible. WZ the sex-chromosome bivalent. NOR ${ }^{S}-$ the short bivalent showing the nucleolus organizer region. $\mathrm{Bar}=5 \mu \mathrm{m} .3 \mathrm{~A}$ detail of the WZ bivalent from the wildtype complement shown in Fig. 2. The longer $\mathrm{Z}$ chromosome axis is twisted along the shorter $\mathrm{W}$ chromosome axis. Arrow indicates the densely staining heterochromatin tangles associated with the $\mathrm{W}$ axis. $\mathrm{Bar}=1 \mu \mathrm{m} .4 \mathrm{An}$ autosome bivalent possessing the knob $(\mathrm{K})$, visible as a band of compact material across the bivalent $[\mathrm{T}(\mathrm{W} ; \mathrm{Z}) 3$ line - female]. $B a r=1 \mu \mathrm{m} .5$ Two nucleolar SCs $\left(\mathrm{NOR}^{\mathrm{L}}\right.$ for the long SC and $\mathrm{NOR}^{\mathrm{S}}$ for the short $\mathrm{SC}$ ) carrying the nucleolus organizer regions, visible as terminal globular structures [ASF-4 line - female $]$. Bar $=2 \mu \mathrm{m}$.

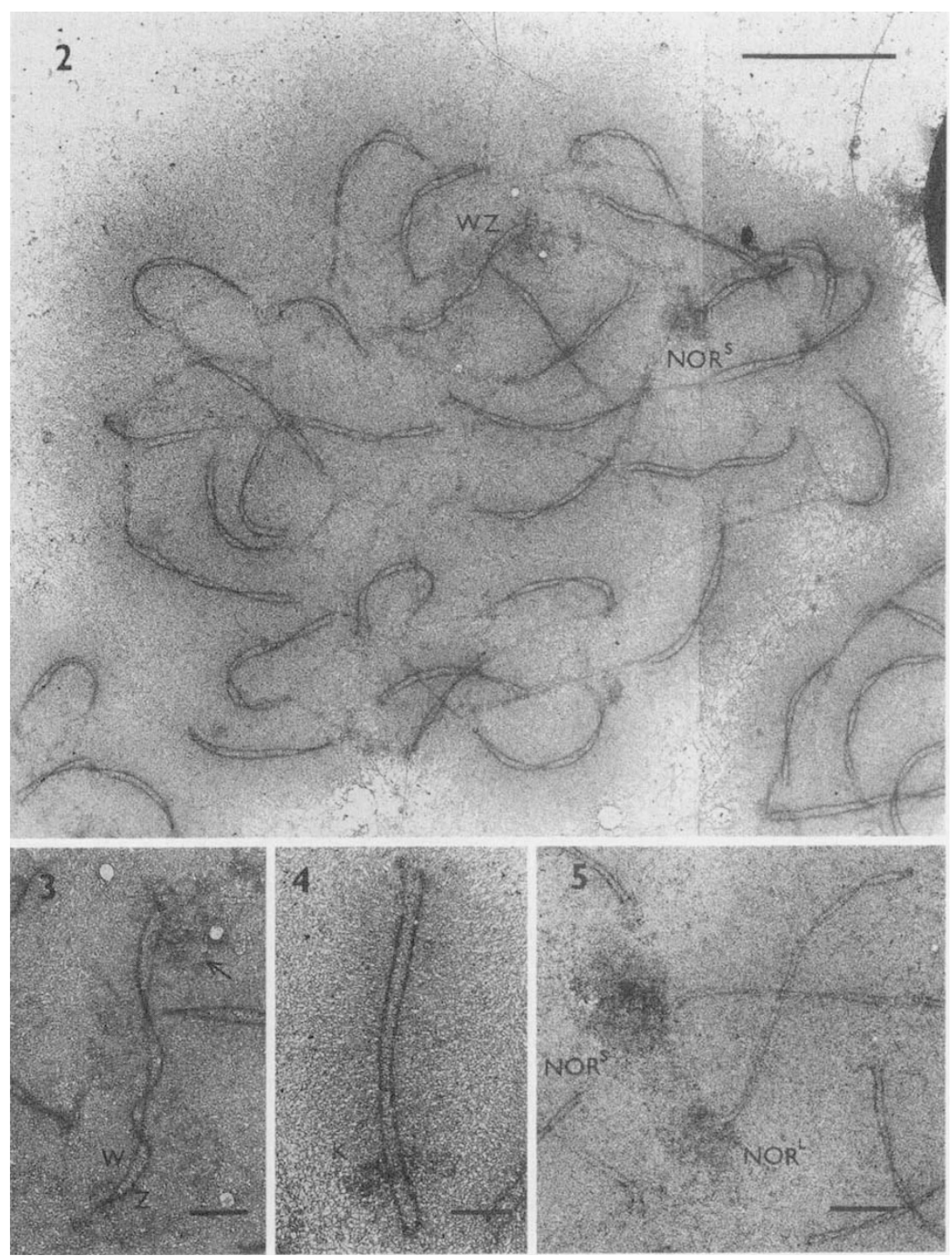

ment lengths; the long $\mathrm{Z}$ LEs measured $2.1 \pm 0.2$ per cent and the shorter W LEs $1.7 \pm 0.2$ per cent. Measurement of sex chromosomes in a few well spread clusters revealed only minor differences among sister nuclei in their lengths (see an example in Table 1) and show that oocyte WZ bivalents do not differ much from nurse cell WZs at that stage. Although the W chromosome in our strain is different (with respect to heterochromatin distribution; unpublished LM observations of $F$. Marec) from that studied by Schulz \& Traut (1979) and Weith \& Traut (1980), their data agree well with ours.

Besides the sex bivalents, three other SCs could be identified in pachytene complements according to their morphology. They occurred in both female and male nuclei of all lines investigated, but were not observed in every pachytene complement probably because of uncontrolled differences in spreading. On one of the 1 st to 5 th longest bivalents, a compact knob appeared besides the homogeneously dispersed chromatin loops (Fig. 4). The compact material formed a $0.5 \mu \mathrm{m}$ (when measured lengthwise) wide band across the SC located about $1 \mu \mathrm{m}$ from the nearest telomere. The knob does not correspond to any particular structure visible under the light microscope (LM) (Schulz \& Traut, 1979). However, the knob bivalent is most probably identical with that observed by Weith \& Traut (1980) in EM microspreads.

Two other bivalents displayed a prominent terminal fluffy globular structure each. The electron dense material of this structure frequently obscured the SC telomeres which it surrounded (Fig. 5). The two SCs 
Table 1 Lengths of the sex chromosome LEs ( $Z$ and $W)$ in eight pachytene nuclei of the same sibling cluster from a wild-type female of E. kuehniella

\begin{tabular}{lllllllll}
\hline & \multicolumn{1}{c}{ Nucleus no. } & & & & & \\
\cline { 2 - 9 } & 1 & 2 & 3 & 4 & 5 & 6 & 7 & 8 \\
\hline Z chromosome $(\mu \mathrm{m})$ & 6.5 & 6.0 & 5.9 & 5.9 & 5.8 & 5.7 & 5.7 & 5.6 \\
W chromosome $(\mu \mathrm{m})$ & 5.5 & 4.8 & 5.1 & 4.8 & 4.7 & 4.7 & 4.6 & 4.9 \\
\hline
\end{tabular}
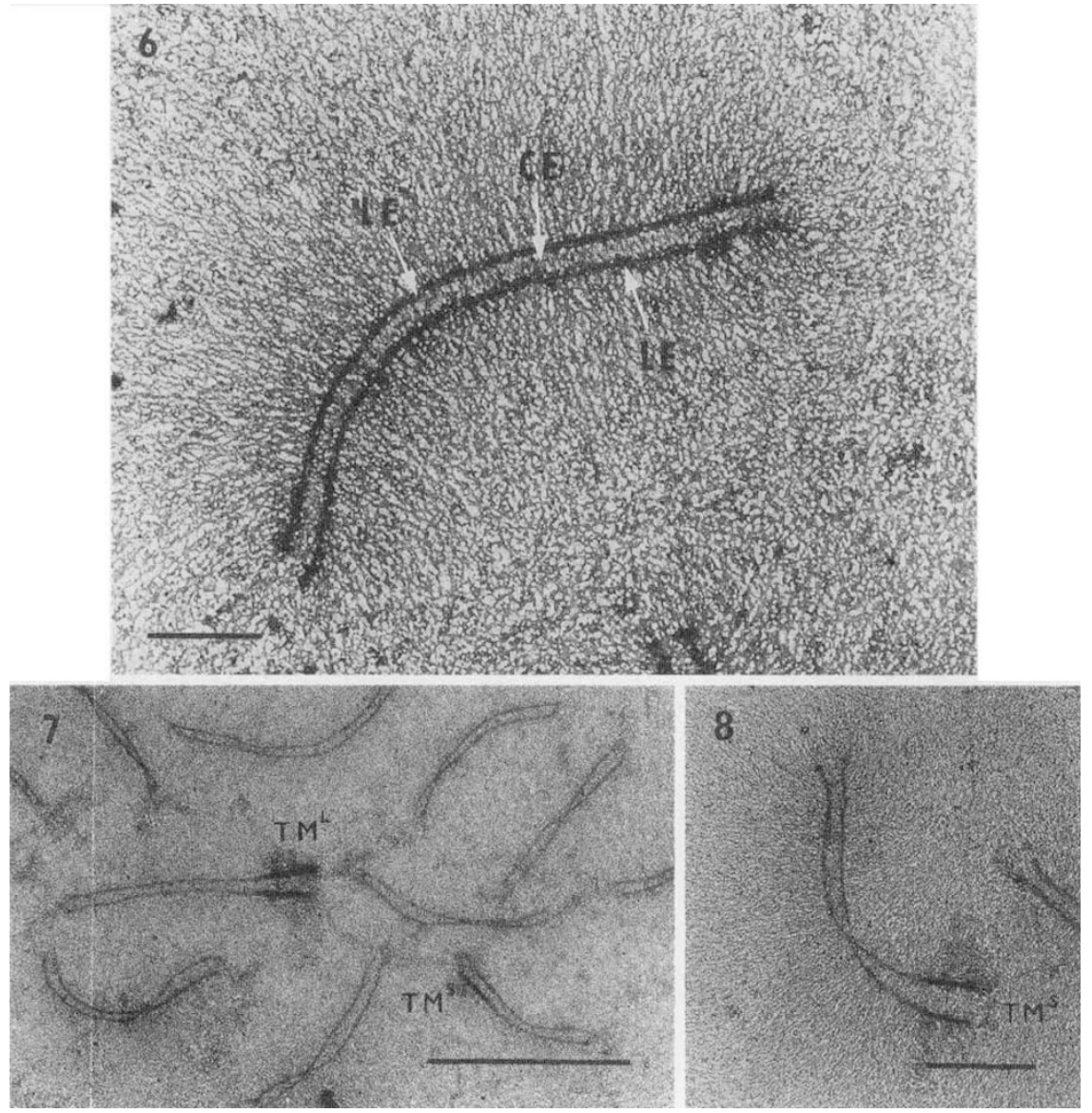

Figs 6-8: 6 The synaptonemal complex of an autosome bivalent from a microspread female pachytene nucleus $[\mathrm{T}(\mathrm{W} ; \mathrm{Z}) 1$ line]. The central element $(\mathrm{CE})$ is visible between two lateral elements (LEs). Chromatin loops radiate from the LEs. Bar $=1 \mu \mathrm{m}$. 7 A part of a female $[T W ; Z) 3$ line] pachytene complement showing two SCs with terminal modifications $\left(\mathrm{TM}^{\mathrm{L}}\right.$ for the long $\mathrm{SC}$ and $\mathrm{TM}^{\mathrm{S}}$ for the short $\mathrm{SC}$ ) of lateral elements. $\mathrm{Bar}=5 \mu \mathrm{m}$. 8 A detail of the short SC displaying the terminal modification $\left(\mathrm{TM}^{\mathrm{S}}\right)$ of lateral elements $[\mathrm{T}(\mathrm{W} ; \mathrm{Z}) 3$ line]. The TM seems to impede full synapsis of the SC. Note the compact chromatin associated with the TM. Bar $=2 \mu \mathrm{m}$. differed significantly from each other by length. The longer one was among the 2nd to 10th longest SCs. The other one represented one of the shortest SCs, at the 24th to 29th position when bivalents were ranked according to their lengths. The two bivalents correspond in length and site of the NOR with the two nucleolar bivalents observed by Schulz \& Traut (1979) in LM preparations stained with lactic acetic orcein. The terminal segments and the attached fluffy globular structures of the two SCs, therefore, are the nucleolus organizer regions (designated $\mathrm{NOR}^{\mathrm{L}}$ for the long $\mathrm{SC}$ and $\mathrm{NOR}^{\mathrm{S}}$ for the short $\mathrm{SC}$ in Fig. 5).

In microspreads prepared from more advanced stages of ovaries, from wandering larvae or prepupae, another morphological marker was observed in a number of pachytene nuclei: a prominent modification of terminal LE segments on one or two SCs (Fig. 7). The modified segments were 2-3 times thicker and more electron dense than normal LE segments and were associated with electron dense chromatin surrounding the terminal modification (TM). In some nuclei, both the SCs showed the TM, in others only one of them. As shown in Fig. 7 (see the short SC), the terminal LE segments were not always symmetrically modified. The chromatin associated with TMs did not exhibit the fibrillar structure typical for spread chromatin loops; it was compact and more electron dense (Fig. 8). Frequently, SCs appeared as if opening 
up in the region of TMs indicating that the modification obstructs perfect synapsis (Figs 7 and 8). We suggest that the two TMs are, in fact, the two NORs $\left(\mathrm{NOR}^{\mathrm{L}}\right.$ and $\left.\mathrm{NOR}^{\mathrm{S}}\right)$ which progressively change their appearance in late pachytene. This suggestion is supported by the following arguments: (i) we never found the terminal globular structures typical for the NOR in nuclei with well developed TMs; (ii) the relative lengths of $\mathrm{NOR}^{\mathrm{L}}$ and $\mathrm{NOR}^{\mathrm{S}}$ bivalents fit very well with those of the long and short TM bivalents, respectively; and (iii) similar LE modifications were observed by Rasmussen (1986) in two SCs, one with a knob and one with the NOR, in late pachytene nuclei of $B$. mori.

\section{Male meiosis}

Male meiotic cells like female cells develop in clusters. Each sibling cluster is connected by a ramified poly- fusome through a system of intercellular bridges (Marec et al., 1993). The clusters are, however, much larger (consisting of 64 primary spermatocytes) and the cells dissociate more easily than the female ones.

The spread pachytene nuclei of wild-type males displayed 30 well paired bivalents (Fig. 9). The sex chromosome bivalent $(\mathrm{ZZ})$ could not be identified due to the perfect homology of the pair and the absence of any other morphological marker. Thus all male SCs appeared similar to the autosomal SCs of females; nevertheless, there was one substantial difference. Almost all male SCs showed one small strongly staining body (Figs 9 and 10, arrows) which we interpret as a recombination nodule ( $\mathrm{RN}$; $c f$. Holm, 1985; John, 1990). The presence of RNs in male SCs and their absence in female SCs corresponds to the fact that male meiosis is chiasmatic, in contrast to the achiasmatic meiosis of Ephestia females. The RNs had a
Figs 9-12 [wild-type strain]: 9 Male synaptonemal complex complement. A total of 30 bivalents is visible. Arrows indicate recombination nodules.

Bar $=5 \mu \mathrm{m} .10$ A male bivalent showing one recombination nodule (arrow). Bar $=1 \mu \mathrm{m} .11$ A detail of a recombination nodule placed in the free space between the two lateral elements. Bar $=500 \mathrm{~nm} .12$ A detail of a recombination nodule placed outside the central region of $\mathrm{SC}$. $\mathrm{Bar}=500 \mathrm{~nm}$.

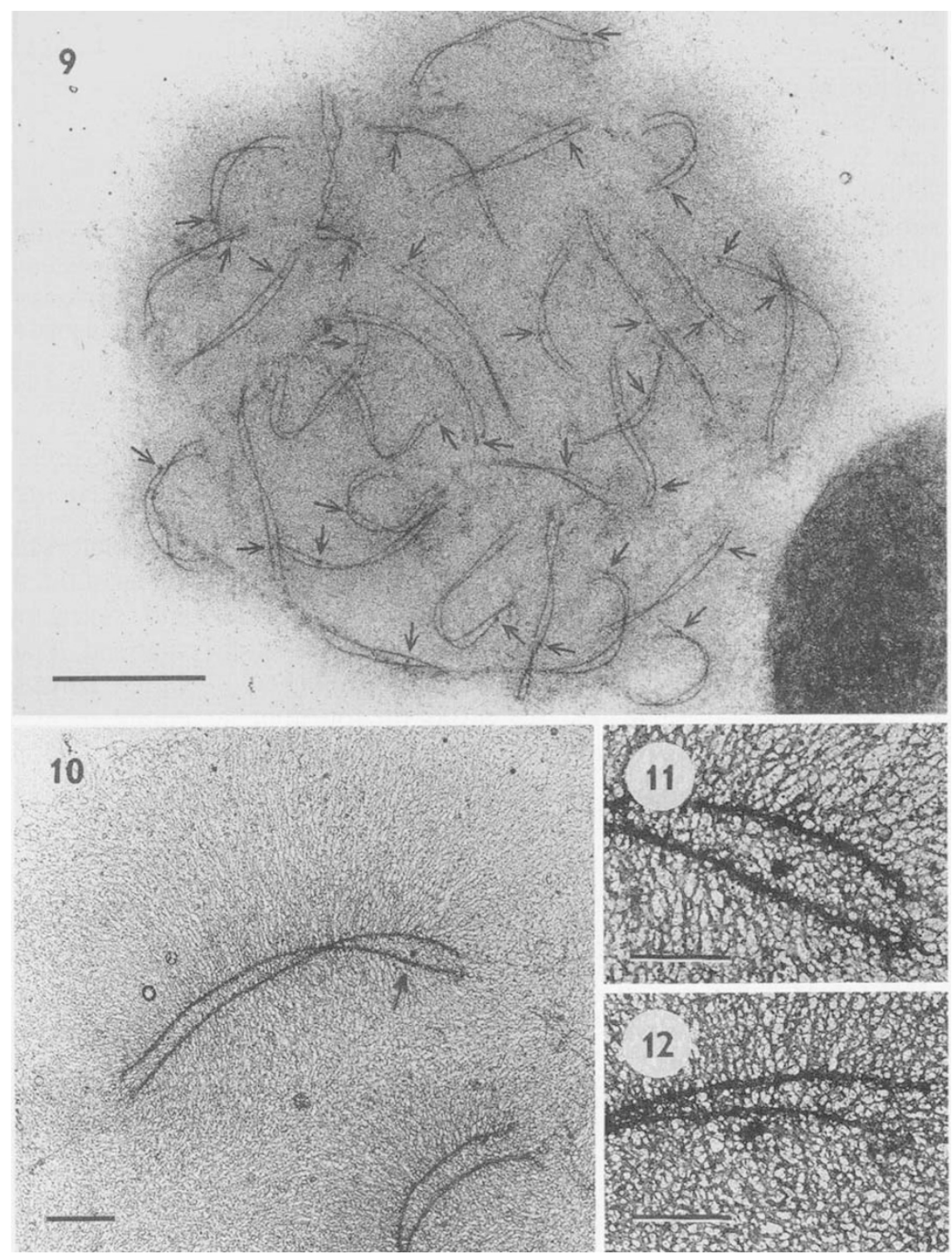


diameter of about $100 \mathrm{~nm}$ and usually appeared like spheres or short bars with an irregular surface. They were placed mostly in the free space between the two LEs (Fig. 11), but sometimes also outside SCs close to one LE (Fig. 12) (cf. Debus, 1978).

To evaluate the number and distribution of RNs, 25 well spread pachytene complements of wild-type males with all 30 bivalents visible in full length were selected. In the 750 bivalents, a total of $767 \mathrm{RNs}$ were recorded (30.7 $\pm 2.2 \mathrm{RNs} /$ nucleus on average). Six hundred and eighty-eight SCs (91.7 per cent) showed one RN, 38 SCs (5.1 per cent) had two RNs and only one SC $(0.1$ per cent) with three SCs was observed. The remaining $23 \mathrm{SCs}$ (3.1 per cent) were devoid of RNs. Since the deviation from a Poisson distribution is highly significant (Chi-square test: $P<0.001$ ) the distribution of RNs among SCs is not random but follows a strict rule.

For the study of the distribution of RNs along bivalents, we were confronted with two obstacles. (i) The three SCs with morphological markers, one with a knob and two with NORs, were recognized only in a fraction of nuclei. (ii) We had no marker for the distinction of the two SC ends. Therefore, we divided each half SC into three regions of equal length (see the schematic drawing in Fig. 13): distal region (D); subdistal region $(\mathrm{S})$; and central region $(\mathrm{C})$. The position of each $\mathrm{RN}$ was recorded and results were statistically evaluated by the Chi-square test. Of the 767 RNs, 348 were located in D, 254 in S and 165 in C positions. These data significantly differ $(P<0.001)$ from the expected equal distribution of RNs. In Fig. 13, the results are expressed as the mean number of RNs in $\mathrm{D}, \mathrm{S}$ or $\mathrm{C}$ positions per nucleus. The figure shows a predominant location of RNs in distal regions of SCs, while location in the central regions was the least frequent.

In contrast to the female complements, we did not find any male complement exhibiting the modified terminal LE segment in the two NOR bivalents. When testes of older male larvae (i.e. the end of the fourth or the beginning of the fifth instar) were used for preparations, many nuclei already showed 30 metaphase I bivalents (Fig. 14). Their chromatin resisted our spreading procedure; it was very condensed and individual chromatin threads were only distinguishable at the surface giving the chromosomes a bristly appearance. Some of the metaphase I bivalents displayed a clear tetrad configuration (Fig. 15) documenting that the four chromatids are already partly separated at this stage ( $c f$. Wolf \& Traut, 1991).

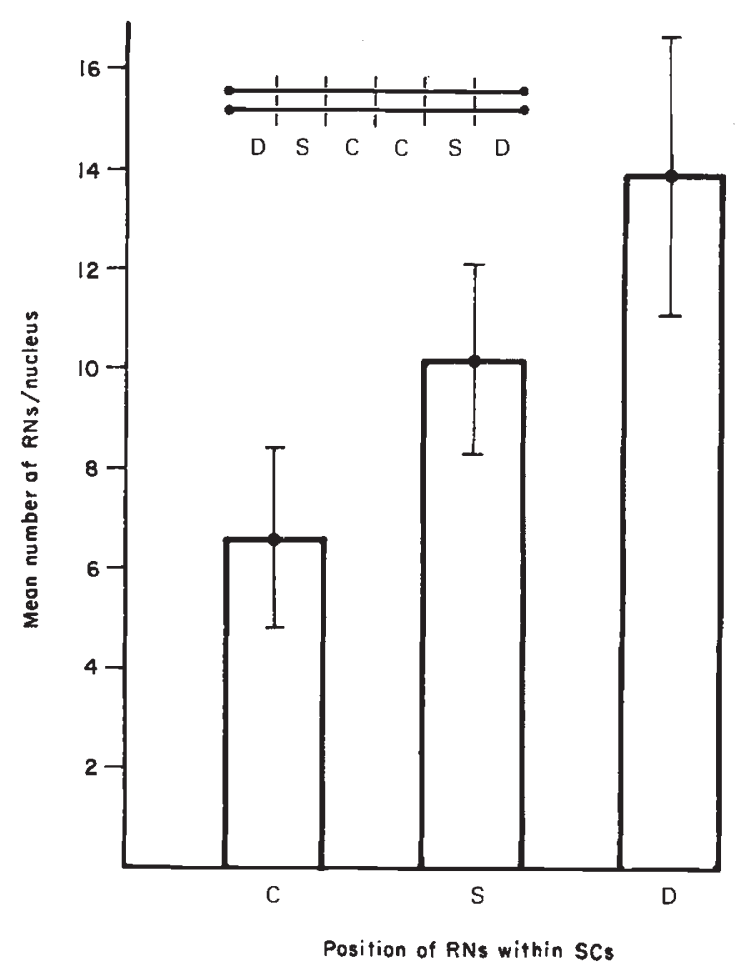

Fig. 13 Frequency distribution of recombination nodules (RNs) along synaptonemal complexes (SCs) in 25 complements of wild-type males. Position of RNs within SCs: C central position, $\mathrm{S}$ - subdistal position, $\mathrm{D}-$ distal position. The positions are illustrated in a schematic drawing of the synaptonemal complex above the diagram.

\section{Chromosome lengths in female and male complements}

Complete sets of 32 female and 31 male pachytene nuclei of the wild-type strain were measured; 13 female and seven male insects contributed to the nuclei analysed. The total lengths of complements (Table 2) varied considerably in both sexes. On average, male complements were longer than female complements $(t$ test; $P<0.05)$. SC lengths within nuclei differed considerably but decreased gradually from the longest to the shortest SCs, and thus it was impossible to use the length as a marker for identification of individual bivalents. The larger the total length of a complement or the average length of the SCs of the complement was, the larger were the differences within the complement. The mean SC length in the shortest female complement was $4.1 \pm 0.8 \mu \mathrm{m}$, but the longest SC ( 5.5 $\mu \mathrm{m})$ was more than twice as long as the shortest one $(2.6 \mu \mathrm{m})$. In the longest female complement, SC lengths varied from 4.5 to $13.6 \mu \mathrm{m}$ (average: $8.1 \pm 2.0 \mu \mathrm{m}$ ). A similar situation was observed in the male complements. SCs in the shortest one measured from 3.6 to 
Figs 14-15: 14 Meiotic metaphase 1 complement of a wild-type male. An equatorial gap, visible in most of the metaphase bivalents, separates two homologous chromosomes. Bar $=5$ $\mu \mathrm{m} .15$ A detail of a metaphase I bivalent from the male complement shown in Fig. 14. A tetrad configuration of this bivalent indicates the formation of four chromatids. Bar $=1 \mu \mathrm{m}$.

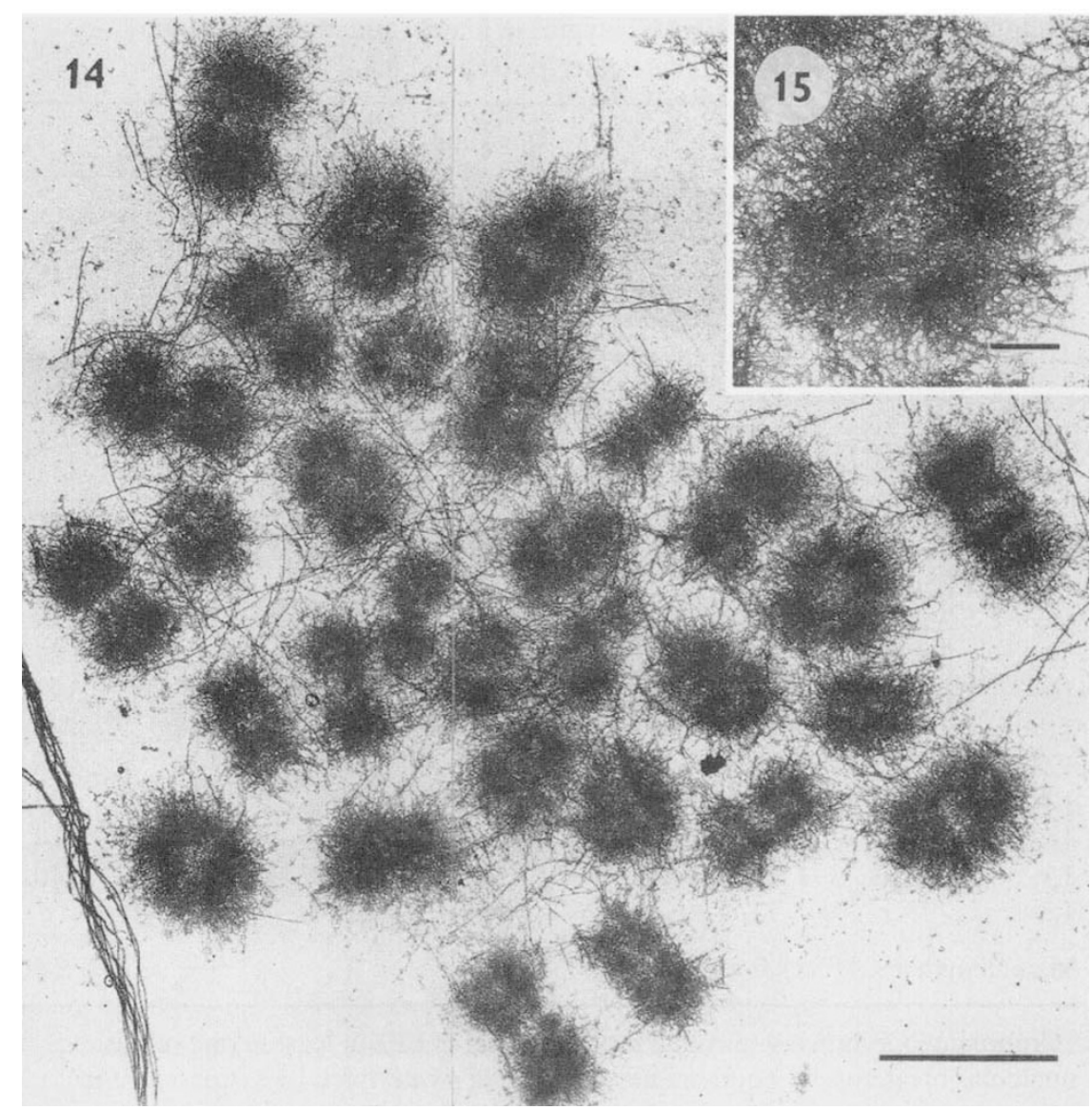

6.6 $\mu \mathrm{m}$ (average: $5.2 \pm 0.9 \mu \mathrm{m}$ ) while in the longest male complement SCs from 5.5 to $12.5 \mu \mathrm{m}$ were observed (average: $9.0 \pm 1.8 \mu \mathrm{m}$ ).

According to Rasmussen (1986), methodological difficulties resulting from the spreading procedure may in part be responsible for the large range and standard deviation of the total LE lengths. Nevertheless, major differences among nuclei might also result from different pachytene substages. The evaluated female and male complements (Table 2), which were randomly selected for measurements, more probably represent a mixture of early, mid and late pachytene nuclei. Three dimensional reconstruction of $B$. mori spermatocytes revealed that the transition from early to mid pachytene is accompanied by a 25 per cent increase in the SC length, and an additional 35 per cent increase appeared from late pachytene to pachytene-diplotene (Holm \& Rasmussen, 1980; see also Table VI in Rasmussen \& Holm, 1979). The staging of sectioned Bombyx spermatocytes was based on SC (bouquet) orientation and the behaviour of centrioles. However, this information is completely unavailable in spread preparations of Ephestia, and therefore, the staging of Ephestia pachytene nuclei is conjectural. On the assumption that the temporal sequence of pachytene events in Ephestia is similar to that of Bombyx, we could roughly distinguish two pachytene substages in spread spermatocytes. Male complements with the total LE length lower than about $400 \mu \mathrm{m}$ (nuclei no. 1-26 in Table 2) probably represented early pachytene nuclei. SCs of these nuclei displayed relatively thick LEs (about 50-60 nm) but CEs were scarcely visible or not visible at all (see Figs 9 and 10). In contrast, male nuclei no. 29 to 31 in Table 2 showed very long and slim SCs with thinner LEs (about 30-40 nm) and with distinct CEs. They were interpreted as mid-late pachytene nuclei.

In female complements, the correlation of the total LE length with the temporal sequence of the SC formation was found to be much more complicated because of achiasmatic meiosis. We tried to use the non-homologous pairing of sex chromosomes as a criterion, however, we did not observe any correlation between the total LE length and degree of $\mathrm{ZW}$ pairing. Complements with the total LE length above $400 \mu \mathrm{m}$ (nuclei no. 25 to 32 in Table 2) displayed, similar to the longest male complements, long and slim SCs with 30-50 nm thick LEs and usually visible CEs (see the two nucleolar SCs in Fig. 5). They might represent mid or mid-late 
Table 2 Total lateral element (LE) lengths in female and male pachytene complements of the E. kuehniella wild-type (ranked data)

\begin{tabular}{|c|c|c|c|c|c|c|c|}
\hline \multicolumn{4}{|c|}{ Female complements } & \multicolumn{4}{|c|}{ Male complements } \\
\hline \multicolumn{2}{|c|}{ Nucleus length } & \multicolumn{2}{|c|}{ Nucleus length } & \multicolumn{2}{|c|}{ Nucleus length } & \multicolumn{2}{|c|}{ Nucleus length } \\
\hline no. & $\mu \mathrm{m}$ & no. & $\mu \mathrm{m}$ & no. & $\mu \mathrm{m}$ & no. & $\mu \mathrm{m}$ \\
\hline 1 & $246.1^{\mathrm{b}}$ & 17 & 298.3 & 1 & 312.1 & 17 & 355.0 \\
\hline 2 & $253.7^{\mathrm{b}}$ & 18 & 299.3 & 2 & 325.3 & 18 & 360.4 \\
\hline 3 & $258.2^{\mathrm{b}}$ & 19 & $300.3^{b}$ & 3 & 326.9 & 19 & 361.3 \\
\hline 4 & $260.4^{\mathrm{b}}$ & 20 & 306.7 & 4 & $327.8^{\mathrm{b}}$ & 20 & 361.4 \\
\hline 5 & $264.6^{\mathrm{b}}$ & 21 & $311.5^{\mathrm{a}}$ & 5 & 329.4 & 21 & 362.4 \\
\hline 6 & $277.7^{\mathrm{b}}$ & 22 & $317.6^{\mathrm{a}}$ & 6 & 331.3 & 22 & 364.1 \\
\hline 7 & 280.7 & 23 & $331.4^{\mathrm{a}}$ & 7 & 334.7 & 23 & 369.9 \\
\hline 8 & 282.9 & 24 & 352.1 & 8 & 336.1 & 24 & 370.0 \\
\hline 9 & 284.6 & 25 & $412.6^{c}$ & 9 & 336.7 & 25 & 387.5 \\
\hline 10 & $285.1^{\mathrm{a}}$ & 26 & $424.1^{c}$ & 10 & 338.7 & 26 & 397.2 \\
\hline 11 & 285.5 & 27 & $427.4^{\mathrm{c}}$ & 11 & 342.8 & 27 & 428.1 \\
\hline 12 & $286.2^{\mathrm{a}}$ & 28 & $455.3^{c}$ & 12 & 345.1 & 28 & 436.2 \\
\hline 13 & $292.7^{a, b}$ & 29 & $459.2^{\mathrm{c}}$ & 13 & 348.5 & 29 & $503.6^{c}$ \\
\hline 14 & 293.2 & 30 & $464.7^{c}$ & 14 & 348.9 & 30 & $514.2^{\mathrm{c}}$ \\
\hline 15 & 293.7 & 31 & $472.1^{\mathrm{c}}$ & 15 & 351.2 & 31 & $540.5^{\mathrm{c}}$ \\
\hline 16 & 295.1 & 32 & $484.7^{\mathrm{c}}$ & 16 & 353.9 & & \\
\hline \multicolumn{6}{|c|}{ Mean length \pm s.d.: $329.9 \pm 74.5$} & \multicolumn{2}{|c|}{$370.6 \pm 56.9$} \\
\hline
\end{tabular}

${ }^{a}$ Complements showing terminal modifications of LEs at least in one of the nucleolar bivalents. ${ }^{b}$ Complements showing SCs with thick LEs (up to $80 \mu \mathrm{m}$ ).

${ }^{c}$ Complements showing long and slim SCs with thin LEs $(30-50 \mu \mathrm{m})$.

pachytene nuclei. On the other hand, extremely short bivalents, which possessed thick LEs (up to $80 \mathrm{~nm}$ ), were found in a number of complements (especially in those of no. 1-6 in Table 2). These complements might belong to nuclei of nurse cells at the end of the pachytene stage, which differentiate after pachytene from oocytes by shortening of bivalents and their subsequent transformation into condensed metaphase-like structures (Traut, 1977; Guelin, 1975). Bivalents of most complements with the total length in the range of 280-350 $\mu \mathrm{m}$ showed, similar to the early pachytene nuclei of males, relatively thick LEs $(50-60 \mathrm{~nm})$ but their other morphological characteristics varied. Several complements displayed TMs or a tendency to TMs in nucleolar bivalents (see Table 2). In most complements, CEs were very thin or absent but in some complements, distinct CEs were observed. Also the degree of ZW pairing markedly varied from unpaired sex chromosomes to perfectly adjusted sex chromosome SCs. Because of these differences, it seems probable that the female complements of medium total LE length include not only early pachytene nuclei but also late pachytene nuclei with SCs gradually shortened after mid or mid-late pachytene stage, and displaying TMs.
Concerning the female complements, similar relations between the SC length and SC morphology were observed in several hundreds of screened nuclei of 12 lines possessing various sex chromosome aberrations. It should also be noted that TMs of the nucleolar SCs were never observed in nuclei showing the long and slim SCs related here to the mid or mid-late pachytene stage.

\section{Discussion}

Comparing female and male meiosis in any lepidopteran species, two phenomena should be taken into consideration. (i) The basic difference in the course of meiotic events derives from the achiasmatic meiosis in females versus the chiasmatic meiosis in males (Suomalainen, 1969b; Traut, 1977; Rasmussen \& Holm, 1982). (ii) There are additional differences resulting from the fact that two types of cells, oocytes and nurse cells, diverge in their development during early oogenesis while all spermatocytes undergo the same sequence of meiotic events (King et al., 1982).

The present study of pachytene complements in $E$. kuehniella revealed that male SCs, in contrast to the female ones, show RNs usually located in a free space 
between the two LEs. The RNs are regularly distributed among SCs with a mean frequency of one RN per bivalent. However, their distribution along SCs is non-random; the RNs are predominantly found in distal regions of SCs. The number and distribution of RNs show good correlation with the number and distribution of chiasmata. No extensive quantitative data are available but according to the cytogenetic study of Traut (1977), bivalents of Ephestia males contain one chiasma each, and many of the chiasmata are distally located.

Holm \& Rasmussen (1980) investigated the occurrence of RNs and chiasmata by means of three dimensional reconstruction of $B$. mori spermatocytes. The number of RNs varied from late zygotene to the end of pachytene. However, both the RNs and chiasmata in Bombyx were at least twice as frequent as those of Ephestia. In contrast to Ephestia, the RNs were randomly distributed not only among but also along bivalents; nevertheless, small deviations from their random distribution among bivalents were revealed at mid and late pachytene. Recently, one or two RNs per SC were reported for males but not for females of another lepidopteran species, the wax moth (Galleria mellonella), using the microspreading technique (Wang et al., 1993). The higher frequencies of RNs in Bombyx and probably also in Galleria correspond to the substantially longer SCs in Bombyx males (Holm \& Rasmussen, 1980) and rather longer SCs in Galleria males (Wang et al., 1993) in comparison with those of Ephestia (this study).

RNs are believed to represent multi-enzyme complexes which mediate meiotic recombination (Holm, 1985; John, 1990; Traut, 1991). Since Lepidoptera females lack crossing over, the presence of $\mathrm{RNs}$ in males of the three lepidopteran species mentioned above and their absence in females together with the excellent correlations found in the present study represent a strong indirect evidence in favour of this hypothesis.

The occurrence of TMs in the two nucleolar bivalents of Ephestia females represents another striking phenomenon in the formation of SCs. The TMs were frequently found in microspreads of the oldest larvae or prepupae, and therefore, it seems probable that they are connected with the late pachytene. The significance of TMs either for the NOR bivalents or for the pachytene stage is not known. Rasmussen (1986) described similar modifications of LEs for two bivalents, one with a knob and one with the NOR, in silver stained spread pachytene nuclei of Bombyx spermatocytes. Since the extent of these modifications increased towards the end of pachytene, he could even use this morphological marker for stage identification. But we did not observe
TMs in any male complement of Ephestia. Either they occur in this species only in females or are limited to a very short period during the SC formation in males, and thus, were not detected.

Concerning the SC lengths, Ephestia females exhibit shorter means than males when the total LE length of pachytene complements is compared. A similar conclusion results from data of sectioned preparations of Bombyx presented by Rasmussen \& Holm (1979). However, this is a rather simplified view of the comparison of SC length between sexes. Our data showed a high variability of the SC length in both sexes. This variability results from major biological differences depending on the pachytene substage, in addition to unknown technical differences due to the spreading procedure. Therefore, mean SC lengths can considerably vary depending on the SC complements selected for measurement. Data on the total complement length in Bombyx showed that in both sexes, the SC lengths increase from early to mid-late pachytene (Rasmussen, 1976; Holm \& Rasmussen, 1980). This increase is especially high in spermatocyte SCs (see Table VI in Rasmussen \& Holm, 1979). A considerable variation in the SC length can also be observed in spermatocytes of Ephestia (this study) which has been interpreted as reflecting similar stage-related evolution of the SC. Regarding changes of the SC lengths in Ephestia females, we suggest that SCs become longer from early to mid or mid-late pachytene but then they are again shortened towards late pachytene as indicated by the appearance of TMs in nuclei with medium total LE lengths. Up to this stage, the oocyte is not differentiated from nurse cells in sibling clusters. Afterwards, the shortening of SCs continue in nurse cells as the extremely short SCs found in some female complements indicate, while the oocyte nucleus forms modified SCs (not observed in this study; see Rasmussen, 1976, 1977). In both sexes of Ephestia, the increase of SC lengths is accompanied by ultrastructural changes of the SC. LEs become thinner and CEs are more distinct ( $c f$. Holm \& Rasmussen, 1980).

\section{Acknowledgements}

We are indepted to Mrs Jutta Leutelt (Lübeck) for her many-sided help with EM procedures and Mrs Ivana Kollárová (České Budějovice) for her help in preparation of the manuscript. This study was supported by a Research Fellowship awarded to F. Marec by the Alexander von Humboldt Foundation (Bonn, Germany). 


\section{References}

DEBus, B. 1978. 'Nodules' in the achiasmatic meiosis of Bithynia (Mollusca, Prosobranchia). Chromosoma, 69, 81-92.

GlaSER, R. W. 1917. Cited in: Lokwood, A. P. M. 1961. 'Ringer' solutions and some notes on the physiological basis of their ionic composition. Comp. Biochem. Physiol., 2, 241-289.

GUELIN, M. 1975. Différenciation de l'ovocyte et des trophocytes au cours de la méiose dans l'ovarie d'Ephestia kühniella Z. (Lépidoptère). C. R. Acad. Sci. Paris, 280, 1091-1094.

HOLM, P. B. 1985. Ultrastructural characterization of meiosis. Biologiske Skrifter(Copenhagen), 25, 39-90.

HOLM, P. B. AND RASMUSSEN, S. W. 1980. Chromosome pairing, recombination nodules and chiasma formation in diploid Bombyx males. Carlsberg Res. Commun., 45, 483-548.

JOHN, B. 1990. Meiosis. Cambridge University Press, Cambridge.

KING, R. C. AND BÜNING, J. 1985. The origin and functioning of insect oocytes and nurse cells. In: Kerkut, G. A. and Gilbert, L. I. (eds) Comprehensive Insect Physiology, Biochemistry and Pharmacology, vol. I, Embryogenesis and Reproduction, Pergamon Press, Oxford, pp. 37-82.

KING, R. C., CASSIDY, J. D. AND ROUSSET, A. 1982. The formation of clones of interconnected cells during gametogenesis in insects. In: King, R. C. and Akai, H. (eds) Insect Ultrastructure, vol. 1, Plenum Press, New York, pp. 3-31.

MANDELBAUM, 1. 1980. Intercellular bridges and the fusome in the germ cells of the cecropia moth. J. Morphol., 166 $37-50$.

MAREC, F. 1990. Genetic control of pest Lepidoptera: induction of sex-linked recessive lethal mutations in Ephestia kuehniella (Pyralidae). Acta Entomol. Bohemoslov., 87, 445-458.

MAREC, F., LEUTELT, J., TRAUT, W. AND WOLF, K. W. 1993. Visualization of polyfusomes in gonads of a moth, Ephestia kuehniella Z. (Lepidoptera, Pyralidae), by a microspreading technique and electron microscopy. Int. J. Insect Morphol. Embryol., in press.

MAREC, F. AND MIRCHI, R. 1990. Genetic control of the pest Lepidoptera: gamma-ray induction of translocations between sex chromosomes of Ephestia kuehniella Zeller (Lepidoptera: Pyralidae). J. Stored Prod. Res., 26, 109-116.

MAREC, F. AND TRAUT, W. 1993. Analysis of structural rearrangements of Lepidoptera chromosomes using the centrifugation spreading technique. In: Kitto, P. H. (ed.) Proc. Int. Symp. Management of Insect Pests: Nuclear and Related Molecular and Genetic Techniques, Vienna, in press.

RASMUSSEN, S. W. 1976. The meiotic prophase in Bombyx mori females analyzed by three-dimensional reconstructions of synaptonemal complexes. Chromosoma, 54, 245-293.

RASMUSSEN, S. W. 1977. The transformation of the synaptonemal complex into the 'elimination chromatin' in Bombyx mori oocytes. Chromosoma, 60, 205-221.
RASMUSSEN, S. W. 1986. Initiation of synapsis and interlocking of chromosomes during zygotene in Bombyx spermatocytes. Carlsberg Res. Commun., 51, 401-432.

RASMUSSEN, S. W. AND HOLM, P. B. 1979. Chromosome pairing in autotetraploid Bombyx females. Mechanism for exclusive bivalent formation. Carlsberg Res. Commun., 44, 101-125.

RASMUSSEN, S. W. AND HOLM. P. B. 1982. The meiotic prophase in Bombyx mori. In: King, R. C. and Akai, H. (eds) Insect Ultrastructure, vol. 1, Plenum Press, New York, pp. 61-85.

RATTNER, J. B., GOLDSMith, M. AND HAMKalo, B. A. 1980. Chromatin organization during meiotic prophase of Bombyx mori. Chromosoma, 79, 215-224.

RATTNER, J. B., GOLDSMITH, M. R. AND hAMKALO, B. A. 1981. Chromosome organization during male meiosis in Bombyx mori. Chromosoma, 82, 341-351.

Robinson, R. 1971. Lepidoptera Genetics. Pergamon Press, Oxford.

SCHUlZ, H. J. AND TRAUT, w. 1979. The pachytene complement of the wildtype and a chromosome mutant strain of the flour moth, Ephestia kuehniella (Lepidoptera). Genetica, 50, 61-66.

SUOMALAINEN, E, 1969a. On the sex chromosome trivalent in some Lepidoptera females. Chromosoma, 28, 298-308.

SuOMalainen, E. 1969b. Chromosome evolution in the Lepidoptera. Chromosomes Today, 2, 132-138.

TELFER, W. H. 1975. Development and physiology of the oocyte-nurse cell syncythium. Adv. Insect Physiol., 11, 223-319.

TRAUT, w. 1977. A study of recombination, formation of chiasmata and synaptonemal complexes in female and male meiosis of Ephestia kuehniella (Lepidoptera). Genetica, 47, 135-142.

TRAUT, w. 1986. A genetic linkage study of W-chromosomeautosome fusions, breakage, and kinetic organization of chromosomes in Ephestia (Lepidoptera). Genetica, 69, 69-79.

TRAUT, w. 1991. Chromosomen. Klassische and molekulare Cytogenetik. Springer-Verlag, Berlin.

TRAUT, W., WEITH, A. AND TRAUT, G. 1986. Structural mutants of the W chromosome in Ephestia (Insecta, Lepidoptera). Genetica, 70, 69-79.

WANG, Y. X., MAREC, F. AND TRAUT, w. 1993. The synaptonemal complex complement of the wax moth, Galleria mellonella. Hereditas, 118, 113-119.

WEITH, A. AND TRAUT, w. 1980. Synaptonemal complexes with associated chromatin in a moth, Ephestia kuehniella Z. The fine structure of the W chromosomal heterochromatin. Chromosoma, 78, 275-291.

WEITH, A. AND TRAUT, w. 1986. Synaptic adjustment, nonhomologous pairing and non-pairing of homologous segments in sex chromosome mutants of Ephestia kuehniella (Insecta, Lepidoptera). Chromosoma, 94, 125-131.

WOLF, K. W. AND TRAUT, w. 1991. Cytology of Lepidoptera. VII. The restructuring of eupyrene prophase I spermatocytes and its relationship to meiotic chromosome and spindle organization in Ephesita kuehniella Z. Protoplasma, 165, $51-63$. 